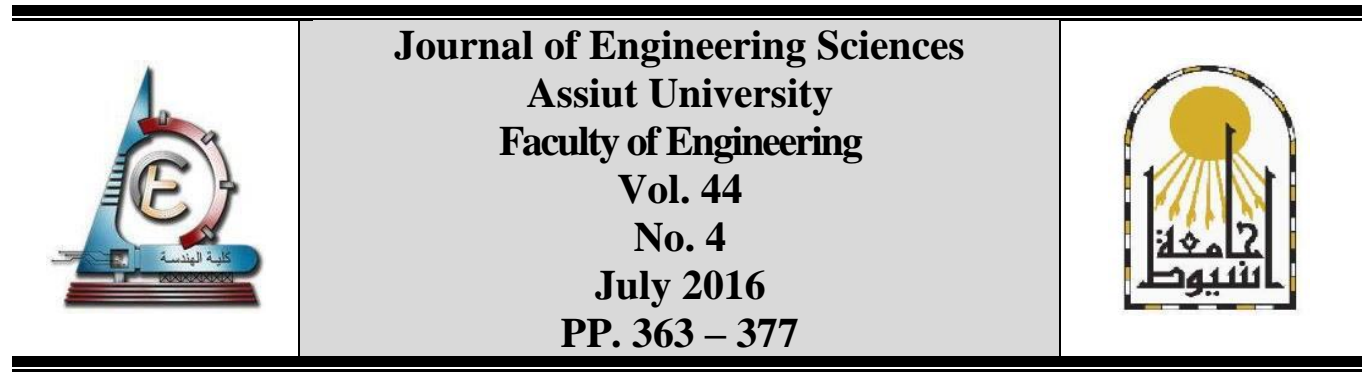

\title{
COMPARATIVE ANALYSIS OF SIMULATION AND OPTIMIZATION TOOLS FOR BUILDING INTEGRATED PHOTOVOLTAICS (BIPV)
}

\author{
Amr Mamdoh Ali Youssef *, 1 , Rabee Mohamed Reffat ${ }^{2}$, \\ Zhiqiang (John) Zhai ${ }^{3}$, Mohamed Abd-ElsamieEid ${ }^{4}$ \\ 1, 2, ${ }_{4}$ Department of Architectural Engineering, Assiut University, Assiut 71518, Egypt. \\ ${ }^{3}$ Department of Civil, Environmental and Architectural Engineering (CEAE), University of \\ Colorado, UCB 428, ECOT 441, Boulder, CO 80309, USA.
}

Received 11 May 2016; Accepted 26 May 2016

\begin{abstract}
A growing attention has been paid to building integrated photovoltaics (BIPV) from both architectural and engineering favorability. There are various computational tools developed to provide computations to optimize BIPVs and often simulations for predicting their performance. This provides a great potential for designers to have different helpful tools to be utilized. This paper introduces a comparative analysis of the most common computational tools that compute or simulate main parameters of BIPVs: building energy consumption, solar exposure radiation flux and PV system performance. These computational tools are classified based the method of processing the inputs and compared using evaluation criteria. Also, optimization algorithms that can be used in optimizing BIPVs have been compared. This comparative analysis helps designers to determine better tool/s and algorithm/s for their design cases and required optimization for BIPV. The main findings of this study are the capabilities, limitations, advantages and disadvantages of each computational tool and optimization algorithm presented, in addition to the best selections among them via a comparative analysis to be used for different design cases.
\end{abstract}

Keywords: BIPVs Computational Tools; Building Integrated Photovoltaics; Building Energy Consumption; Incident Solar Exposure Flux; PV Systems performance.

\section{List of Abbreviations}

PV $\quad$ Photovoltaics

BIPV Building Integrated Photovoltaics

DOE-2 A simulation engine version 2 released by Department $\underline{\text { Of }}$ Energy , US [18]

SAM System Advisor Model (a software tool) [19]

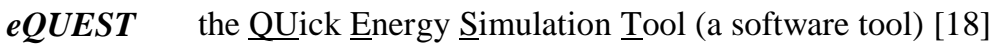

* Corresponding author.

E- mail address: amr.ma.youssef@gmail.com 
JES, Assiut University, Faculty of Engineering, Vol. 44, No. 3, May 2016, pp. 363 - 377

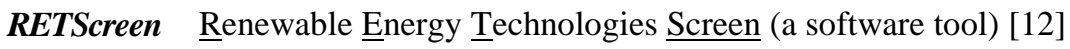

CAD Computer-aided design

VR4PV Virtual Real-time simulation of irradiance for photovoltaic (PV) products [9]

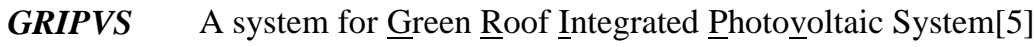

ZEBO a design decision making tool for Zero Energy residential Buildings in hot climates [16]

BEopt A Software for Building Energy Optimization [20]

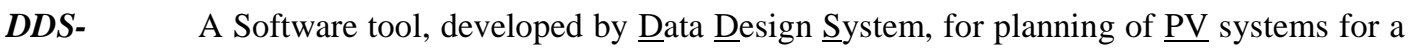

CADPV number of applications using $\underline{\mathrm{CAD}}[\overline{13}]$.

GUI Graphical $\underline{\text { User }}$ Interface

$\boldsymbol{G A} \quad$ Genetic $\underline{\text { Algorithm }}$

\section{Introduction and background}

Building integrated Photovoltaic (BIPV) refers to photovoltaic (PV) modules that are integrated with the building through its envelope as a part of the building architectural design. PV modules in BIPV represent one of the main architecture features. BIPV is one of the most recent applications that received growing attention due to both architectural and engineering favorability. BIPV provides a great potential of utilizing solar radiation to generate portion of the needed electricity for buildings through wide facades. Accordingly, BIPV cover a higher percentage of the energy needs in buildings, especially in high-rise buildings that have a limited roof [1]. For example, ASHRAE standard specifies the consumption of a commercial building in hot climates to be $13.4 \mathrm{kWh} /\left(\mathrm{ft}^{2} . \mathrm{yr}\right)$, while it is estimated that roof-mounted PV can generate $40.4 \mathrm{kWh} /\left(\mathrm{ft}^{2} . \mathrm{yr}\right)$ in standard test conditions ${ }^{1}$, which merely supplies 3 floors with electricity in average ${ }^{2}[2]$. Therefore, there is a need to integrate PV modules in building facades. Architecturally, BIPVs provide wider varieties of facades and flexible envelope especially with various BIPV systems that are available in today's markets.

A wide variety of computational tools are available to study the optimization of PV integration in buildings and simulate different sides of its performance. This number of tools provides a great potential to the designers to study their BIPV designs from different perspectives. However, there still needs to specify which of this tool/s is better for such a design to perform the required optimization. This paper presents a comparative analysis of current computational tools of BIPV, classifies them and carries out a detailed comparative analysis between them. The tools have been studied, analyzed and classified based on three main parameters: building energy consumption, solar radiation flux and PV system performance.

\section{Computational tools in BIPV}

There are various computational tools simulate and optimize the BIPV performance. These computational tools can be classified mainly based on their methods of processing the inputs to produce outputs to two groups: Computational tools for building envelope and

${ }^{1}$ Standard test conditions: 1000 watts per square meter of sunlight intensity and hold a cell temperature of $25^{\circ} \mathrm{C}$ [2].

2 The indicated numbers are approximate averages (per the unit area) specified by ASHRAE standard mainly for generic high-rise commercial buildings in hot climates; and calculated based on the standard electrical loads required for them [2]. 
simulation tools for evaluating BIPV performance. Computational tools for building envelope: these tools can handle with a given building envelope and provide a generation of surfaces, forms, alternatives, optimizations or others as produced outputs; the required calculations or simulations are performed inside. These tools achieve different BIPV optimizations for the inputted building, not only simulations for a current situation. Evaluation and simulation tools: these tools simulate and evaluate the BIPV performance, and provide detailed analysis for surface temperature, radiation, building energy consumption, PV systems energy generation, PV systems' sizing and/or other parameters (e.g. payback period, costs, etc).These tools are used mainly in the evaluation process of an existing design BIPV envelope or system. However, they cannot provide new generations/solutions for given cases as other computational tools for BIPV envelope which is the main difference between both groups. Detailed analysis of each group, related tools and parameters in each tool are addressed in the following sections.

\subsection{Computational tools for building envelope}

In the literature, there are many computational tools for building envelope, which are generating new solutions or alternatives. Topaloğlu [3] used "SOLVELOPE" computer program to generate a 3D volume for the envelope to satisfy the annual needs from solar energy in a given site. SOLVELOPE conducts the calculations needed to protect the site or the area that is placed on the northern side of a given site, and generates a 3D volume that satisfy the annual needs from solar energy in the site. Capeluto et al [4] developed "SUSTARC" as a computational tool to evaluate a given building or site configurations from the concept of "solar rights" ensuring solar access to each building on site and neighboring buildings. However, two outputs can be generated: a) the solar rights envelope which represents the maximum heights that allow the solar rights of any existing buildings inside or outside the site; and b) the solar collection envelope which represents the lowest possible locations of windows and passive solar collector that is not shaded. Also, Sui and Munemoto [5] developed "GRIPVS"as a simulation methodology to achieve the optimal shape of the green gable roof for best $\mathrm{CO}_{2}$ emission and investment value. This includes evaluations of the special single slope roof on which a green lawn is laid and PV systems are installed. The generated roof (as one of the output) has the optimal area ratio, roof ridge position and pitch of PV roof slope. Yezioro [6] developed "PASYS" (a CAD tool) to evaluate the best-suited thermal comfort design strategies (passive systems type and size) for a given building with providing design guidelines for improvements. Although it doesn't provide new building envelope designs or generations of alternatives, it generates design guidelines through evaluating facades for better envelope performance. The outputs are presented in a text file that includes recommendation of suitable passive systems, and determination of effective southern facade area (in northern hemisphere) that allows maximum deviation adequacy. Kampf and Robinson [7] developed "RADIANCE" as a computational program that optimizes urban geometric forms for the utilization of solar irradiation. It is also integrated into the simulation workflow to measure daylighting. However, the main outcome of RADIANCE is

\footnotetext{
${ }^{3}$ The concept of "solar rights" in urban design refers to allow passive heating of buildings in winter to improve the comfort conditions of people in streets, sidewalks and open spaces. Since people have the right to enjoy sun benefits, the building and space design that doesn't consider this concept may cause uncomfortable conditions inside [4].
} 
three available shapes: a) project envelope as one block, b) project envelope as number of blocks, and c) roof shape (geometry). Each of these outputs is supported by a rendered image and a chart for the irradiation on grid points divided on building surfaces. In addition to the generated building detail, shape, dimensions, volume are also produced.

Moreover, Veiga and Roche [8] developed "EvSurf" as an internet-based program to evaluate and analyze the relationships of solar position with envelope surfaces. This is achieved through evaluating the potentials of solar radiation on surfaces. EvSurf determines the effects of direct solar radiation in complex building envelope and complex surfaces in different orientations, and presents the capability to calculate the irradiance, shading, temperature surfaces and PV system performance. The main output of EvSurf is a graphical visualization of rating the possible solar radiation values with the surface area. This can be used for optimizing envelope surfaces via provided virtual 3D environment in a HTML window. Veldhuis and Reinders [9] established"VR4PV" software tool to be used for evaluating the distribution of irradiance on specific building envelope surfaces with an arbitrary geometry that can be covered with PV cells. VR4PVestimates the energetic performance during the design process of BIPV, and supports the preliminary layout selection of PV modules in the whole design. However, the building has to be imported from a CAD tool including the 3-D surroundings, and accordingly it allows shadow calculation for multiple surrounding objects with various shapes. Its output includes geometries with the irradiance on their oriented surfaces to be covered with PV modules.

Autodesk ECOTECT [10] is a whole-building energy analysis tool; it simulates solar radiation on each surface, shading of surfaces, thermal comfort, building energy consumption and others. Also, it generates best alternatives of shading devices or solar envelope for a specific case, and allows generations of building envelope based on solar rights' concept (ensuring solar access to each building on site and neighboring buildings).This is achieved through performing the required calculations on a given building, generating related alternative/s and providing a visual color scale of solar exposure on surfaces of the given building and its alternatives. However, building models can be developed in ECOTECT or imported as a CAD file (computer-aided design), and the model components (roofs, walls, windows, doors and etc) are then identified automatically via the virtual 3D Environment. The outputs of ECOTECT include simulation results (graphs, tables, xls sheets, etc) for solar radiation, thermal analyses, shading and others. It can be used mainly to optimize BIPVs through analyzing solar exposure on their surfaces [11].

\subsection{Simulation tools for evaluating BIPV performance.}

Energy simulation tools are useful for evaluation purposes as they provide help in taking decisions about the quantitative and economic appropriateness of designed systems, without generating new solutions (the main difference between computational and simulation tools as illustrated before). Therefore, energy simulation tools are generally performed inside the computational tools for BIPV envelope; this is to provide internal evaluations for the generated alternatives they produce. It will be useful in this paper to analyze some of the most common energy simulation tools, since there are a huge number of tools validated by international agencies for their various purposes.

There are various simulation tools that focus on building energy consumption or PV system performance. For example, Renewable Energy Techniques Screen (RETSCREEN) tool is one of the most common simulation tools in this field for PV sizing and testing. It is an Excel-based 
analysis software tool that can provide a simulation of the energy generation, cost and required payback period of the PV system. Such tools help decision makers quickly and inexpensively to determine the technical and financial viability of potential renewable energy, energy efficiency and cogeneration projects [12]. DDS-CADPV [13] was developed to enable the planning of PV systems for a number of applications; the roof, façade or ground mounted PV systems can be planned, visualized, documented in 2D and 3D preview. It freely defines cross-sections, part models and elevations for different models and allows detailed assembly plans, complete bills of material, system diagrams and reports. EnergyPlus [14] is a whole building energy analysis software that enables building professionals to simulate a building design. It can produce detailed tables, charts and information for the whole energy performance of pre-designed PV systems. EnergyPlus provides diverse outputs such as analysis and simulations of a given building along with related systems (including PV systems), electricity end use and others. These outputs are in different formats (.xls, HTML, IDF and others) which can be easily used with other platforms. Therefore, many simulation tools were developed using the source engine of EnergyPlus for their simulations, but with an easier Graphical User Interface (GUI) provided for primary users. They focus only on specific purposes and/or better 3D visualization. For example, "Open Studio" is a free plug-in developed as a toolbox in SketchUp [15].This plug-in is for visualizing and editing the building geometry using the SketchUp interface. For, example, solar exposure can be visualized in the sketchUp 3D environment with visual color scale, while surfaces can be classified automatically to types (e.g. roofs or walls, openings or solids, high or low solar exposure, etc) in the same interface. Also, the simulation details of these outputs can be extracted as an IDF file (EnergyPlus format). "ZEBO" [16] is a decision tool that facilitates and integrates the use of energy performance simulation using EnergyPlus engine. It was developed to be used during the design development in the early design phase of residential buildings, and PV system can be planned, visualized, documented in 2D and 3D. The tool estimates the average performance of a PV system in different locations in the hot humid climate.

Also, Simergy [17] is a comprehensive GUI for EnergyPlus that presents and visualizes energy simulation for variations of a baseline model, and the geometry of the site contents can be created, edited and presented in reports through different formats(IFC, IDF, SIMP and others). Results include a summary of: project details, site consumption, zone simulations, lighting and daylighting, HVAC sizing summary and others. DesignBuilder [18] provides indepth analysis of energy use and consumption of any building design using EnergyPlus. It provides an advanced modeling from the concept to completion. The outputs of the whole building simulations and calculations are presented in tables and charts. DOE-2 [18] is a widely used and accepted building energy analysis program for predicting the energy use and cost for all types of buildings. It performs a detailed comparative analysis of building designs and technologies via text files. The inputs can be an inp files that contain: building dimensions, orientation, wall details, system details and others; while the outputs are tables and numbers for the building energy consumption graph, HVAC systems' performance and others. For easier use, eQuest [18] was developed using the engine of DOE-2, in order to visualize the designed model in a 3D environment with an easier-to-use GUI.

Other simulation tools were developed with focusing on detailed features of PV systems' performance not buildings, which can be used for studying PV systems in general and BIPVs in particular, however, they can still contribute in a limited room in BIPV 
design studies. System Advisor Model (SAM) [19] is a simulation tool for simulating PV modules when attached to building surfaces in different orientations, and its outputs are the PV system generation, payback period and others for each integration position and case. It has a friendly easy-to-use GUI that shows results for monthly energy output, life-cycle cost chart, cost analyses through reports, tables and graphs. Many other tools are simulating the PV systems' performance without any significant difference (just different interfaces or different details' presentation), such as PV-DesignPro, PVSYST [19], while others are focusing on the cost analysis of energy use. For example, BEopt [20] is a tool to identify cost-optimal alternatives of house energy use for a design. It identifies the near optimal design alternatives from the benchmark to achieving zero net energy houses using EnergyPlus engine, and it provides detailed simulation-based analysis based on specific house characteristics, such as size, occupancy, vintage, location and utility rates. The main outputs include tables for horizontal, direct and diffuse irradiation on surfaces in addition to detailed hourly output for any user-selected building design. These outputs can be presented in different formats (XML, CSV, XLS, IDF files and others).

Besides the introduced main differences that classified the tools to two major groups (Computational tools for building envelope and simulation tools), all the introduced tools have also major differences and classifications either in purposes or computational capabilities. The analysis of these tools based on their main purposes various differences were revealed. For example, SOLVELOPE, RADIANCE and Autodesk ECOTECT optimize 3D geometries for the utilization of solar irradiation using different concepts, while SUSTARC, EvSurf and VR4PV provide only limited optimizations/evaluations for a given envelope from different solar perspectives. Some tools can be considered as decision tools such asPASYS and ZEBO to be used in evaluating building energy consumption. Others are mainly for simulating the PV systems generation and require details numerically, such as RETSCREEN, SAM, PV-DesignPro, PVSYST and others, while DDS-CADPV can instead visualizes PV systems for a number of applications in $2 \mathrm{D}$ and 3D preview along with providing the relevant details. Few tools are only limited to specific design cases and details, such as BEopt that identifies cost-optimal alternatives of house energy use and GRIPVS that generates the optimal shape of the green gable roof for best $\mathrm{CO}_{2}$ emission and investment value. By analyzing the general computational capabilities of the introduced tools, major differences were identified. For example, some tools have a 3D environment such as SOLVELOPE, RADIANCE, EvSurf, VR4PV and others, while some tools are very easy-to-use compared to others such as Open Studio Plug-in and SAM. Few can be considered as engines to be easily interfaced through or integrated in other systems such as eQuest (DOE-2) and EnergyPlus, while others are not a stand-alone systems (relevant to other engines) such as Open Studio Plug-in. Few tools can be communicated (their outputs can be used as inputs in other tools) using similar file extensions, such as EnergyPlus, Open Studio Plug-in, Simergy and BEopt via .idf files.

Therefore, there is a need to analyze and compare these tools, since each one has different inputs, internal processes, limitations, capabilities, advantages and disadvantages for conducting different outputs. Also each tool can import and export different file extensions (.txt, .idf, .jpg, etc).This comparative analysis is required for designers to determine which combination/s of tools are more useful for their BIPV design cases. In the following section, computational tool are classified, and accordingly compared, based on 
Amr Mamdoh Ali Youssef, comparative analysis of simulation and optimization tools for building ...

their main outcomes to simulate BIPVs parameters, also more details about each tool and its detailed differences compared to others are provided.

\section{Analysis of the current computational and simulation tool in BIPV}

For analyzing the current both computational tools for building envelope and simulation tools in studying BIPVs performance, the main parameters that will be focused/measured in this paper are: a) building energy consumption: the net energy consumption in the building is the main parameter that is used for studying buildings in general and analyzing their integration with PV modules in specific as it provides a complete perspective about the building optimization rather than only maximizing the PV systems generation of integrated systems; b) solar radiation flux: this parameter is one of the main parameters affecting either PV systems generation or building energy consumption for BIPV designs, and it focuses on testing the effect of orienting, moving and tilting PV modules integrations in building surfaces; and c) PV system performance: this parameter includes the different output of PV systems integrations and relevant optimizations.

Based on each of the previous parameters, the following software packages shown in Table 1 will be compared. Accordingly, the capabilities of these tools in simulating and assessing each of these parameters were compared as will be presented in the following sections.

\section{Table 1.}

An overall comparative analysis software packages of the most common computational tools for building and energy simulations

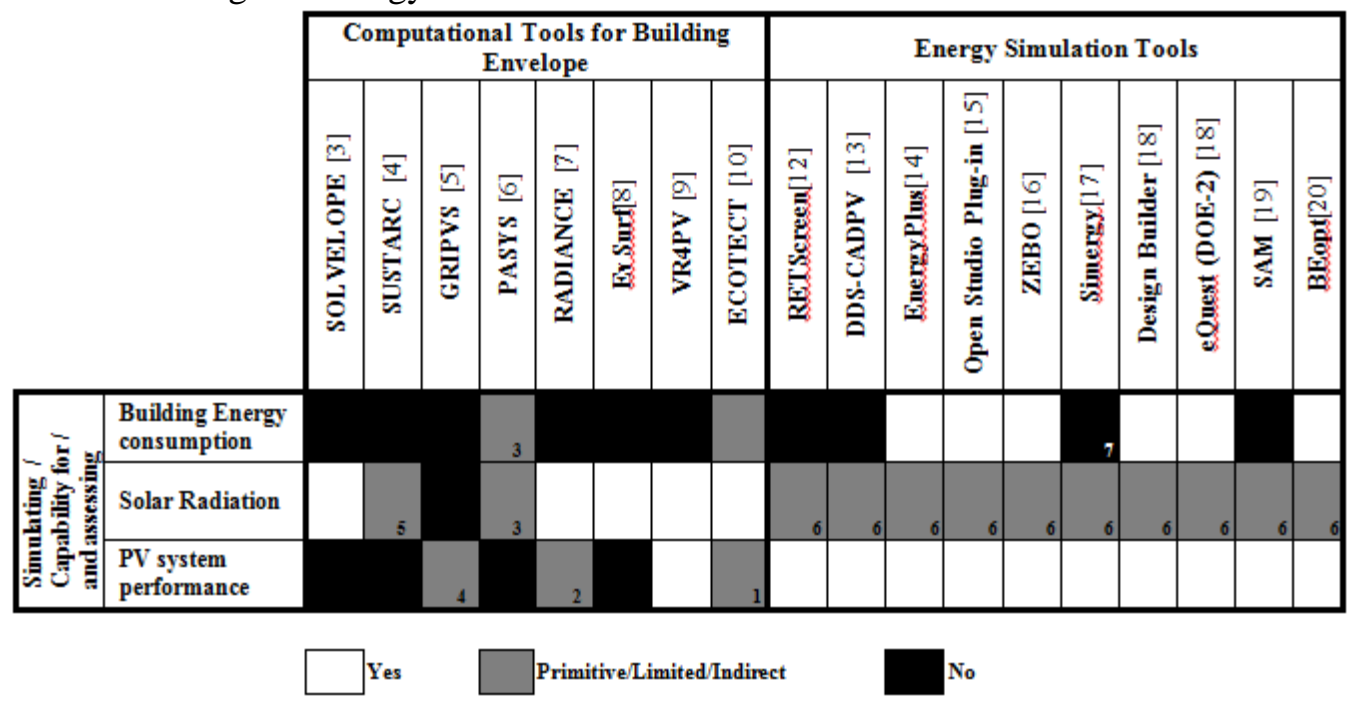

\section{Notes:}

1. PV system performance can be evaluated indirectly through solar exposure.

2. PV systems are provided only for few applications.

3. Sets only appropriate thermal comfort strategies and design guide lines for improvement [6], primitive measurements are provided.

4. Evaluates $\mathrm{CO}_{2}$ emissions and investment values of PV systems in gable roofs. 
5. Generates and evaluates different building configurations that ensure solar rights [4].

6. Shading (as neighbors' effect) is calculated and considered in building energy consumption simulation.

7. Only main components (HVAC, Water loop, etc) are included.

\subsection{Analysis of tools for simulating building energy consumption}

Starting from the building energy consumption as a parameter, the tools that mainly simulate and/or assess energy consumption as shown in Table 1 are EnergyPlus, Open Studio plug-in, BEopt, Design Builder, ZEBO and eQuest (DOE-2). In order to compare these tools, a set of capabilities and limitations have been identified, such as the capability to simulate custom shapes instead of basic ones, generic building types instead of specific ones, building surroundings instead of a main building only. In addition, a set of computational advantages were also included for conducting the comparative analysis, such as being easy-to-use and friendly Graphical User Interface (GUI), etc. Table 2 illustrates a comparative analysis between the computational tools for simulating building energy consumption based on the above capabilities and computational advantages.

Based on the conducted analysis, EnergyPlus and eQuest (DOE-2) tools are the best tools that satisfy the capability criteria, and accordingly are used by designers for simulating buildings energy consumption in different design cases. Both can be interfaced and accordingly integrated in the other platforms. eQuest has a 3D environment that can be utilized to visualize building models, while Energyplus uses other 3D interfaces (such as SketchUp) to visualize 3D models. eQuest is an easy-to-use compared to EnergyPlus and requires less computing time [21]. With avoiding the capability to interfacing the tools easily, DesignBuilder and Open Studio Plug-in can be used as some of the best options. Other tools have more limitations that may prevent its use completely in specific design cases. For example, BEopt can be used only for residential houses that are less than 4 floors [20], while ZEBO is limited to a generic rectangular single-zone template [16].

\subsection{Analysis of tools for simulating solar exposure}

Some of the most common tools that mainly simulate and/or assess solar exposure are ECOTECT, VR4PV, EvSurf, RADIANCE and SOLVELOPE. The previous set of capabilities, limitations and computational advantages is used for conducting a comparative analysis as shown in Table 3. ECOTECT and RADIANCE have satisfied the majority of criteria, accordingly they are better than others to be used in different generic design cases from simulating solar exposure, while others have different limitations, such as Solvelope is only for sites, not buildings.

\subsection{Analysis of tools for simulating $P V$ system performance}

Some of the most common tools that mainly simulate and/or assess PV performance are VR4PV, EnergyPlus, Open Studio, BEopt, Simergy, DesignBuilder, RETScreen, ZEBO, DDS-CADPV, SAM and eQuest (DOE-2). Some of the previous comparison criteria have been used in this comparative analysis, while other criteria have been included to address the purpose of simulating PV performance, such as containing editable and flexible PV systems as inputs (not only few specific modules or features), in addition to providing detailed analysis for designed PV performance as outputs. Table 4 illustrates a detailed comparative analysis of tools simulating PV system performance. DDS-CADPV, VR4PV and SAM have satisfied the majority of the capabilities and computational advantages, and accordingly can be used in generic design cases. They also have either a large set of PV modules or flexible inputs to be 
Amr Mamdoh Ali Youssef, comparative analysis of simulation and optimization tools for building ...

inserted if a specific PV module is required to be applied. However, other tools have limitations, such as VR4PV that requires a 3D model from CAD to be imported first [9].

\section{Table 2.}

A detailed comparative analysis of most common computational tools for simulating and assessing measure or simulate building energy consumption

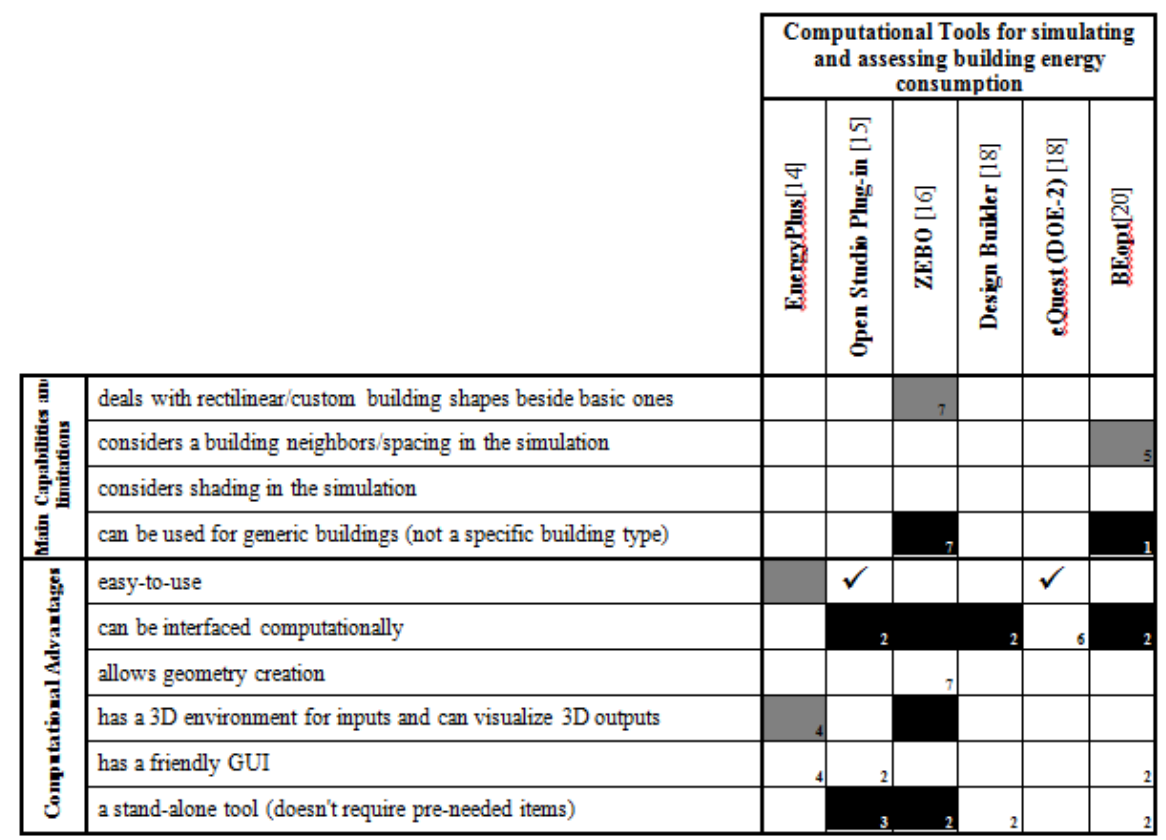

\begin{tabular}{|l|l|l|l|l|l|l|}
\hline Best tools based on capabilities and computational advantages & $\checkmark$ & & & & $\checkmark$ & \\
\hline
\end{tabular}

$\checkmark$ The best regarding this item $\square$ Yes $\square$ Lack/ Limited/ Partial Ind irect

\section{Notes:}

1. PV system performance can be evaluated indirectly through solar exposure.

2. PV systems are provided only for few applications.

3. Sets only appropriate thermal comfort strategies and design guide lines for improvement [6], primitive measurements are provided.

4. Evaluates $\mathrm{CO} 2$ emissions and investment values of PV systems in gable roofs.

5. Generates and evaluates different building configurations that ensure solar rights [4].

6. Shading (as neighbors' effect) is calculated and considered in building energy consumption simulation.

7. Only main components (HVAC, Water loop, etc) are included.

\section{Analysis of optimization algorithms for optimizing BIPVs}

The need for optimization algorithms in BIPV field is to find optimal values of building envelope through the extensive number of possible BIPV solutions, which are conducted through different variables (e.g. length, width, height, WWR, etc.) and their internal options (e.g. length has an acceptable range in each case). For instance, WWR, as one variable, can vary to 9 options (10\% to $90 \%$ ) for each façade, and combining only these options in a simple L-shape building can produce 531,441 possible solutions (options ^ 
facades $=9 \wedge$ 6)for evaluation, and adding other variables could extend the possible solutions to millions. Hence, this number of solutions cannot be evaluated with the normal methods to select best ones or the optimal one since this requires an inapplicable high computational time. Therefore, an optimization algorithm is necessary to be used for identifying the best combinations of BIPVs variables and options. Optimization algorithms are generally solving problems related to optimization through finding a solution with the highest achievable performance under given constrains, and specifically are trying to find the minimum values of mathematical functions [22]. In the current field, this mathematical function could be, for instance, minimizing the energy consumption of buildings, minimizing PV payback period or others. These objective functions should be outlined with specific limitations (e.g. variables, options, etc.).

There are various optimization algorithms available that can be used. For instance, Genetic Algorithm (GA) uses a cycle of random exploration that leads to successive reproduction of global solutions. So, GA can avoid a local maximum or minimum if the population finds better values in other definition domain areas. Moreover, GA performs well in difficult types of functions, such as linear, non-linear, continuous, discontinuous functions and others. GA performs with large ensembles, complex problems and large number of probabilistic variables. GA optimization starts with conducting a population of random individual solutions; each solution is a combination of options selected from different variables (chromosomes), and each solution has a fitness function (a value) based on evaluation criteria to be optimized. Best solutions in each population can be repeatedly optimized towards better populations using two GA operations: crossover and mutation. The crossover operation produces two new solutions by combining two groups of options in the two previous solutions, and the mutation operation creates a solution by changing an option or more in one solution. These operations lead to more successful solutions in each population. However, GA is one of the most common algorithms that have been used in solving optimization problems [23, 24, 25].

Hill Climbing is an optimization technique for solving computationally hard problems. It starts with an arbitrary solution to a problem, then attempts to find a better solution by making a local/small modification in a part (a single element) of a solution towards better one. Hill Climbing requires only a limited amount of memory for efficient implementation, but it is disabling to detect the insolvability of a problem instance, so several solutions will be conducted for just better solutions [26]. Simulated Annealing is a local search metaheuristic used to address discrete, extend continuous optimization problems, and incorporate sub-ways together based on their size of change. The incorporation is accepted based on a formal parameter. Although it can optimize functions that are not defined for some parameter values, but it is completely memory-less as it disregards all historical information gathered during the algorithm's execution [26]. Tabu Search uses the concept of memory by controlling the algorithm's execution via a list of forbidden moves. A Tabu list is created for the most recent local modifications to solutions, in order to be utilized in following solutions and to be repeated again. This list is updated with each modification, but if the number of iterations is very large, computing time will be increased [27].

Ant Colony is a probabilistic technique and heuristic method for solving computational problems to find good paths through graphs. It searches for optimal path/s in the graph based on the behavior of ants, which are seeking a path between their colony and source of food. Whenever it is using a small number of individual classifiers, it will be one of the 
best algorithms to be used. It is based on the probability of each part of the best path to the solution, so the efficiency of sub-connections cannot be judged [28]. Greedy Algorithm is an algorithm that always takes the best local solution while finding an answer; this is achieved via working in different phases, and it finds the best local solution that can be achieved at each phase, and then chooses the best local optimum to start from. Accordingly, the search ends up at a global optimum [29]. Harmony Algorithm is conceptualized meta-heuristic algorithm that uses the musical process of searching for a perfect state of harmony, this is through trying to find a vector in variables to optimize a certain objective function; each variable generates a value for finding a best global optimum all together. Harmony Algorithm also uses a stochastic random search instead of a gradient search so that derivative information is unnecessary [30].

Table 5 illustrates a comparative analysis of the introduced optimization algorithms using their main capabilities and limitations. All of them except "Hill Climbing" can find global solutions through large ensembles, while GA, Tabu Search, Simulated Annealing and Ant Colony can deal with different complex optimization problems. Regarding the time of optimization, Ant Colony represents one of the most time-consuming algorithms compared to others, and the optimization is not certain in some cases (exactly like Hill Climbing). The conducted research related BIPVs show that the most common algorithms used in optimizing buildings in general and solving similar architectural problems to BIPVs in particular are respectively GA, Simulated Annealing then Ant Colony. However, GA and Simulated Annealing can achieve the specified capabilities to be used in optimizing BIPVs.

Table 3.

A detailed comparative analysis of most common computational tools $t$ for simulating and assessing solar exposure

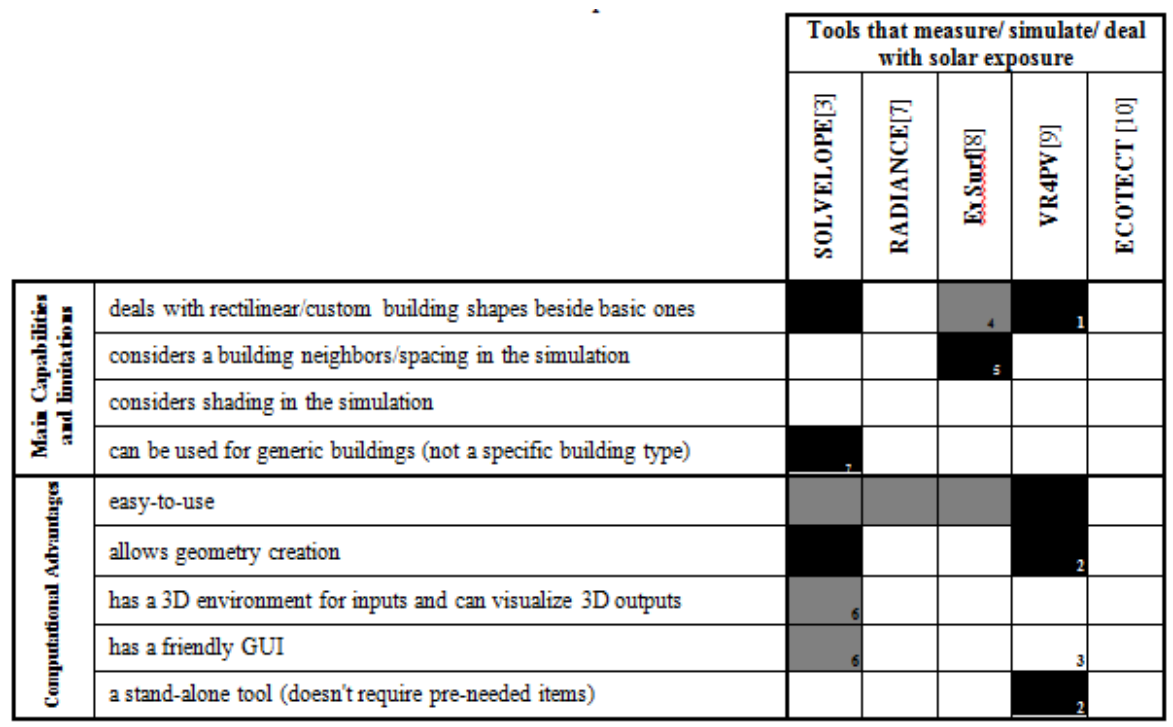

Best tools based on capabilities and computational advantages

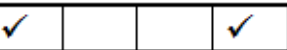




\section{Related notes:}

1. Only for tilted surfaces.

2. Needs a 3D CAD geometry as an input.

3. Has lack of a GUI.

4. Generates tilted surfaces.

5. Only performs one building.

6. Needs numerical inputs to be converted by the tool to 3D outputs in a poor $3 \mathrm{D}$ environment. for flat rectangular sites, not buildings.

\section{Conclusion}

This paper presented a comparative analysis for the common computational tools related to BIPVs performance, which can be studied using three parameters: building energy consumption, solar exposure and PV system performance. First, most common computational tools related to the BIPV field were collected, studied and analyzed. Essentially, these tools can be classified in two groups based on methods to process outputs and type of outputs: computational tools for building envelope and energy simulation tools. Tools in both groups have been compared again based on the introduced three comparison criteria namely building energy consumption, solar exposure and PV system performance. Accordingly, relevant tools to each of the comparison criteria have been compared based on their different capabilities and computational advantages, so the best tool/s have been determined to be used in different design cases, in addition to tool limitations for relevant specific purposes.

As a result, tools that mainly simulate and/or assess building energy consumption are EnergyPlus, Open Studio plug-in, BEopt, Design Builder and eQuest (DOE-2). EnergyPlus and eQuest (DOE-2) tools were found to be the best tools that satisfy the majority of the comparison criteria, and accordingly are used by designers for simulating building energy consumption in generic design cases. Other tools have more limitations that limit its use completely to specific design cases such as BEopt; it is for residential houses. Also, tools that mainly simulate and/or assess solar exposure are ECOTECT, VR4PV, EvSurf, RADIANCE and SOLVELOPE. However, ECOTECT and RADIANCE were found to be better than others in their use in different generic design cases for simulating solar exposure. While others have different limitations, such as Solvelope; it is only used for sites, not buildings. On the other hand, tools that simulate and/or assess PV system performance are VR4PV, EnergyPlus, Open Studio, BEopt, Simergy, DesignBuilder, RETScreen, ZEBO, DDSCADPV, SAM and eQuest (DOE-2). DDS-CADPV, VR4PV and SAM were found to be better than others in their use in generic design cases as also they have a large set of PV modules or flexible inputs to be inserted for a specific PV module. However, other tools have limitations, such as VR4PV requires a 3D model from CAD to be imported first. Also, Genetic Algorithm (GA) and Simulated Annealing search can achieve the specified capabilities to optimize BIPVs, while conducted research related to BIPVs uses GA, Simulated Annealing then Ant Colony in solving similar architectural problems.

The presented review and analysis of computational tools relevant to BIPVs can be useful for designers in providing it helps to determine better tools for their relevant design cases and required purposes in energy simulation, along with identification of main limitations of one of these tools. This comparative analysis can be further extended in the future to include other parameters to BIPVs, such as thermal insulation and ventilation of PV systems distributed on BIPV facades. Adding the architectural perspective of BIPVs 
may require involving different design concepts and tools which can be used in designing BIPVs. This will provide a comprehensive guide to BIPVs designers and researchers that can be used widely in different design cases.

\section{Acknowledgements}

The authors would like to acknowledge the financial support of the Egyptian Ministry of Higher Education for this research.

\section{REFERENCES}

[1] EIA (2013) International Energy Statistics, Electricity Generation. Available via: http://www.eia.gov/tools/faqs. Accessed at 16 Oct. 2014.

[2] ASHRAE (2007). ANSI/ASHRAE/IESNA Standard 90.1-2007. Final Qualitative Determination.

[3] Topaloğlu, B. (2003). Solar envelope and form generation in architecture. Master Thesis, Middle East Technical University, Turkey. Available via: https://etd.lib.metu.edu.tr/upload/1042704/index.pdf. Accessed at 20 March 2016.

[4] Capeluto, G., Yezioro, A., Bleiberg, T. and Shaviv, E. (2005). From computer models to simple design tools: Solar rights in the design of urban streets. Proceedings of $9^{\text {th }}$ IBPSA International Conference, Montreal, Canada.

[5] Sui, J. and Munemoto, J. (2007). Shape Study on a Green Roof Integrated Photovoltaic System for Bi-objective Optimization of Investment Value and $\mathrm{CO}_{2}$ Emission. Asian Architecture and Building Engineering 6: 307-314.

[6] Yezioro, A. (2009). A knowledge based CAAD system for passive solar architecture. Renewable Energy 34(3): 769-779.

[7] Kampf, J.H. and Robinson, D. (2010). Optimization of building form for solar energy utilization using constrained evolutionary algorithms. Energy and Buildings 42: 807-814.

[8] Veiga, J.D. and Pablo, L. (2011). A Computer Solar Analysis Tool For The Design And Manufacturing Of Complex Architectural Envelopes: EVSURF, Urbanism Environmental Models and Geographic Information Systems: 105-109. Available via: http://cumincad.scix.net/data/works/att/3183.content.pdf. Accessed at 20 March 2016.

[9] Veldhuis, A.J. and Reinders, A.H.M., (2012). Real-Time Irradiance Simulation for PV Products and Building Integrated PV in a Virtual Reality Environment. IEEE Journal of Photovoltaics 2(3): 352-358. Available via: http://ieeexplore.ieee.org/stamp/stamp.jsp?tp=\&arnumber=6185630\&isnumber=6220282. Accessed at 20 March 2015.

[10] Autodesk (2014). ECOTECT Analysis Software Homepage. Available at http://usa.autodesk.com/ecotect-analysis. Accessed 20 Oct. 2012.

[11] Youssef, A.M.A., Zhai, Z., Reffat, R.M. (2015). Design of Optimal Building Envelopes with Integrated Photovoltaics. Building Simulation, Online first articles. Available via: http://link.springer.com/article/10.1007/s12273-015-0214-y. Accessed at 18 March 2016.

[12] NRCAN (2014) Natural Resources Canada, RETScreen International. Available via http://www.retscreen.net/ang/home.php. Accessed at 20 Oct. 2014.

[13] DDS (2013). Data Design System. A Nemetschek Company. Available via: http://www.dds-cad.net/company/. Accessed at 20 March 2016.

[14] EERE (2014).EnergyPlus Energy Simulation Software, Energy Efficiency \& Renewable Energy, US Department of Energy. Available via: http://apps1.eere.energy.gov/buildings/energyplus. Accessed at 5 Nov. 2014. 
[15] Open studio (2014) OpenStudio 1.5.0 Release. Available via https://www.openstudio.net. Accessed at 20 Oct. 2014.

[16] Attia S (2012). A Tool for Design Decision Making-Zero Energy Residential Buildings in Hot Humid Climates. PhD Thesis. UCL, Belgium. Available via: http://www.shadyattia.net/research/phd.html.Accessed at: 20 March 2016.

[17] EERE (2014). Simergy, Energy Efficiency \& Renewable Energy, US Department of Energy. Available via: http://simergy.lbl.gov/index.html. Accessed at 20 March 2016.Gondhalekar, A.C., Petrov, E.P. and Imregun, M. (2009). Parameters Identification for Nonlinear Dynamic Systems Via Genetic Algorithm Optimization. Journal of Computational and Nonlinear Dynamics 4(4): 2-9.

[18] DOE-2 (2014) DOE-2 based software homepage, eQuest. Available via http://www.doe2.com/equest. Accessed at 20 Oct. 2014.

[19] NREL (2014) System Advisor Model, SAM. Available via https://sam.nrel.gov. Accessed at 20 Oct. 2014.

[20] NREL (2015) BEopt. Available via https://beopt.nrel.gov/home. Accessed at 20 March 2016.

[21] Rallapalli, H.S. (2010). A Comparison of EnergyPlus and eQUEST Whole Building Energy Simulation Results for a Medium Sized Office Building. M.Sc. Thesis, Arizona State University, US. Available via: https://repository.asu.edu/attachments/56303/content/Rallapalli_asu_0010N_10220.pdf.Ac cesssed at: 20 March 2016.

[22] Hardesty, L. (2015). Optimizing optimization algorithms - Analysis shows how to get the best results when approximating solutions to complex engineering problems. MIT News. MIT University, US. Available via: http://news.mit.edu/2015/optimizing-optimizationalgorithms-0121. Accessed at: 20 March.

[23] Mardle, S. and Pascoe, S. (1999). An overview of genetic algorithms for the solution of optimization problems. CHEER 13(1): 16-26. Available via https://www.economicsnetwork.ac.uk/cheer/ch13_1.Accessed at 20 March 2016.

[24] Gondhalekar, A.C., Petrov, E.P. and Imregun, M. (2009). Parameters Identification for Nonlinear Dynamic Systems via Genetic Algorithm Optimization. Journal of Computational and Nonlinear Dynamics 4(4): 2-9.

[25] Jin, J.T., Cho, H.J. and Jeong, J.W. (2012).Optimization of Freeform Building Shape Using Genetic Algorithm. Proceedings of the $1^{\text {st }}$ Asia International Building Performance Simulation Association (IBPSA) Conference and Exhibition (ASim2012), Shanghai, China.

[26] Selman, B. and Gomes, C.P. (2000). Hill Climbing Search. Encyclopedia of Cognitive Science: 333-335. Available via: http://www.cs.cornell.edu/gomes/selman-gomes-encyclhillclimbing.pdf. Accessed at: 20 March 2016

[27] Lim, A., Rodrigues, B. and Zhang, J.(2005) Tabu Search Embedded Simulated Annealing for the Shortest Route Cut and Fill Problem. Journal of the Operational Research Society. , 56(7), 816. http://ink.library.smu.edu.sg/lkcsb_research/2621.Accessed at 20 March 2016.

[28] Shekhawat, A., Poddar, P. and Boswal, D. (2009) Ant colony Optimization Algorithms: Introduction and Beyond. Indian Institute of Technology Bombay Artificial Intelligence Seminar. Available via: http://mat.uab.cat/alseda/MasterOpt/ACO_Intro.pdf. Accessed at: 20 March 2016.

[29] Levitin, A. (2012). Introduction of The Design and Analysis of Algorithms. Pearson Education. Available via: www.vgloop.com/_files/1394454921-126688.pdf. Accessed at 20 March 2015

[30] Wang, X., Gao, X. and Zenger, K. (2015) An Introduction to Harmony Search Optimization Method, SpringerBriefs in Computational Intelligence: 5-11. Available via: http://link.springer.com/book/10.1007\%2F978-3-319-08356-8. Accessed at 20 March 2016. 


\section{تحليل مقارن لأدوات تحسين ومحاكاة المباني المدمجة بالخلايا الثمسية}

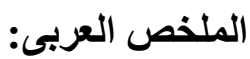

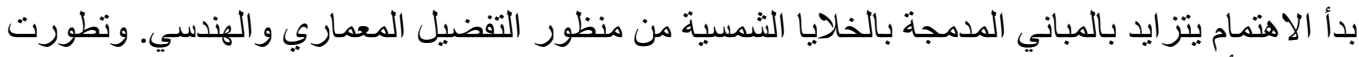

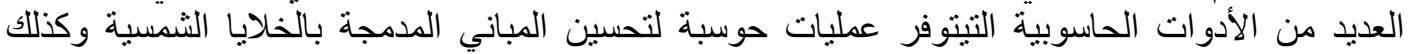

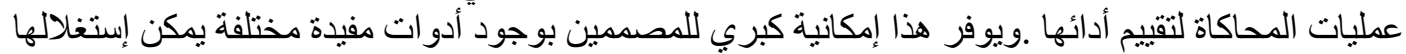

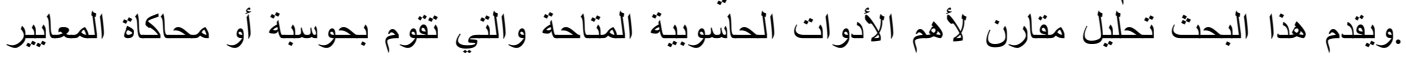

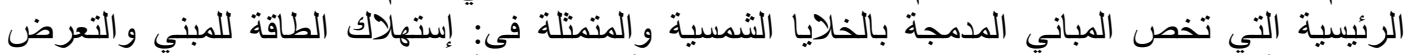

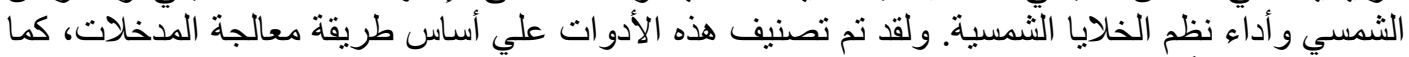

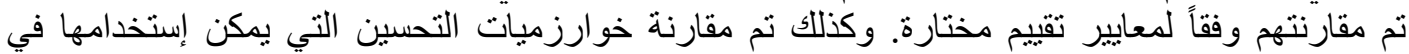

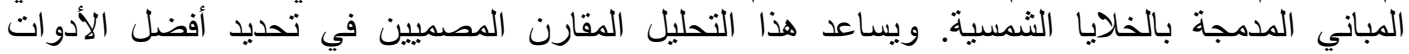

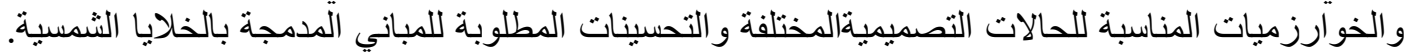

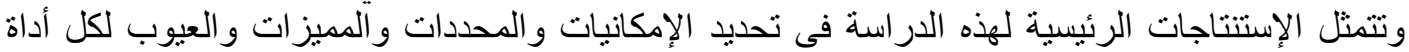

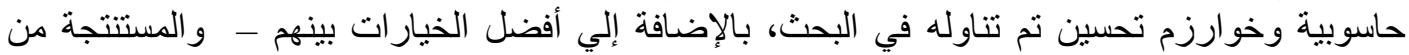
التحليل المقارن - في الحالات التصميمية المختلفة.

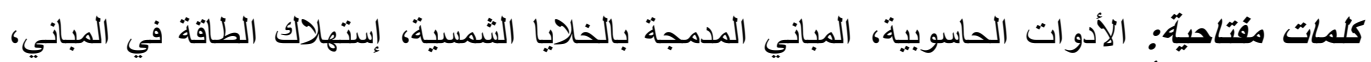
التعرض الثمسي، أداء نظم الخلايا الثنمسية. 